\title{
Intermittent Dialysis Leading to Dialysis Disequilibrium Syndrome
}

\author{
Kalyani Regetia , Waqas Jehangir ${ }^{\mathrm{a}, \mathrm{c}}$, Sherry Kumar ${ }^{\mathrm{b}}$, Chadrick Chua ${ }^{\mathrm{a}}$, \\ Abdalla Yousif ${ }^{\text {a }}$, Shuvendu Sen ${ }^{\mathrm{a}}$
}

\begin{abstract}
Dialysis disequilibrium syndrome (DDS) is a critical and ultimately fatal condition that presents in hemodialysis patients. Serious manifestations of DDS involve impaired concentration, disorientation, and coma. Risk factors for the condition include dialysis treatment, elevated BUN, renal disease, metabolic acidosis, and pre-existing neurologic disease. There are three main theories proposed to account for the development of DDS, including a reverse urea effect, idiogenic osmoles, and paradoxical brain acidosis. Each of these theories potentiates cerebral edema, and eventually brain herniation, that leads to death. This case examines a 33-year-old Hispanic male brought to the emergency room. He was admitted to the ICU based on hospital findings and had a metabolic acidosis that persisted despite appropriate initial treatment. He was eventually placed on hemodialysis over the course of 2 weeks, and ultimately expired from DDS. This paper aims to demonstrate that hemofiltration is a superior alternative in patients where hemodialysis is indicated, so that DDS, and subsequent fatality, can be avoided.
\end{abstract}

Keywords: Dialysis disequilibrium syndrome; Herniation; Hemodialysis

\section{Introduction}

Dialysis disequilibrium syndrome (DDS) is a rare and serious complication of hemodialysis that manifests with neurological symptoms including impaired concentration, hallucinations, disorientation, and coma, if left untreated. These neurological symptoms are primarily attributed to cerebral edema [1]. Furthermore, brain death can eventually ensue and become fatal. DDS can present after receiving first time dialysis treatment,

Manuscript accepted for publication July 15, 2015

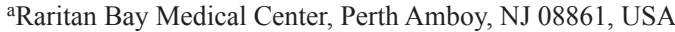

bRoss University School of Medicine, Miramar, FL, USA

${ }^{\mathrm{c} C o r r e s p o n d i n g ~ A u t h o r: ~ W a q a s ~ J e h a n g i r, ~ R a r i t a n ~ B a y ~ M e d i c a l ~ C e n t e r, ~} 530$

New Brunswick Ave, Perth Amboy, NJ 08861, USA.

Email: wjehangir@hotmail.com

doi: http://dx.doi.org/10.14740/wjnu228e or intermittent dialysis. First time hemodialysis patients, especially those with markedly elevated blood urea nitrogen levels, are at greatest risk for DDS [1].

\section{Case Report}

A 33-year-old Hispanic male was brought to the hospital by emergency services after he was found unconscious at home. At the time, the patient's vitals included heart rate of $74 / \mathrm{min}$, respiratory rate of $26 / \mathrm{min}$, and a systolic blood pressure of 80 $\mathrm{mm} \mathrm{Hg}$. His pupils were round and reactive to light. The remainder of his physical examination was unremarkable.

Initial laboratory investigations revealed a white blood cell count of $24.7 \times 10^{3} / \mathrm{mm}^{3}$, blood urea nitrogen of $17 \mathrm{mg} /$ $\mathrm{dL}$, serum creatinine of $3.5 \mathrm{mg} / \mathrm{dL}$, anion gap of 27 , arterial blood gases $\mathrm{pH}$ of $6.95, \mathrm{PaCO}_{2} 79 \mathrm{~mm} \mathrm{Hg}, \mathrm{PaO}_{2}<37 \mathrm{~mm}$ $\mathrm{Hg}, \mathrm{HCO}_{3} 17.4 \mathrm{mmol} / \mathrm{L}$, and lactate $24 \mathrm{mmol} / \mathrm{L}$. These findings were consistent with increased leukocytosis, high anion gap metabolic acidosis, and respiratory acidosis. The metabolic acidosis was partially accounted for by acute renal failure with retained unmeasured anions and ketonemia. Liver function tests further demonstrated elevated ALT and AST. Creatinine kinase and CKMB were also found to be elevated. Toxicology reports and urine drug screen were positive for cocaine and opiates. Urinalysis was negative for infection causing organisms. Electrocardiogram showed normal sinus bradycardia (Fig. 1). Computed tomography (CT) of the head was negative for intracranial bleed and did not show any anoxic encephalopathy (Fig. 2). The patient had intact gag and cough reflexes. Based on laboratory and radiographic findings, the patient was admitted to the ICU with a provisional diagnosis of cardiopulmonary arrest secondary to drug overdose, acute kidney failure, and septic shock. The patient was empirically started on vancomycin and piperacillin and tazobactam.

Despite being placed on a sodium bicarbonate drip (150 $\mathrm{mEq}$ of $8.4 \%$ sodium bicarbonate in $1000 \mathrm{D} 5 \mathrm{~W})$, his metabolic acidosis persisted. He was further given fluid resuscitation of about $3 \mathrm{~L}$, and still remained oliguric. A decision was made by the patient's nephrologist to start hemodialysis. The sputum culture showed many Streptococcus serogroup $\mathrm{C}$ and urine culture showed Klebsiella pnuemoniae ESBL. During a hospital stay of approximately 14 days, the patient received dialysis

Articles @ The authors | Journal compilation @ World J Nephrol Urol and Elmer Press Inc ${ }^{\mathrm{TM}} \quad$ | www.wjnu.elmerpress.com

This is an open-access article distributed under the terms of the Creative Commons Attribution License, which permits unrestricted use, distribution, and reproduction in any medium, provided the original work is properly cited 


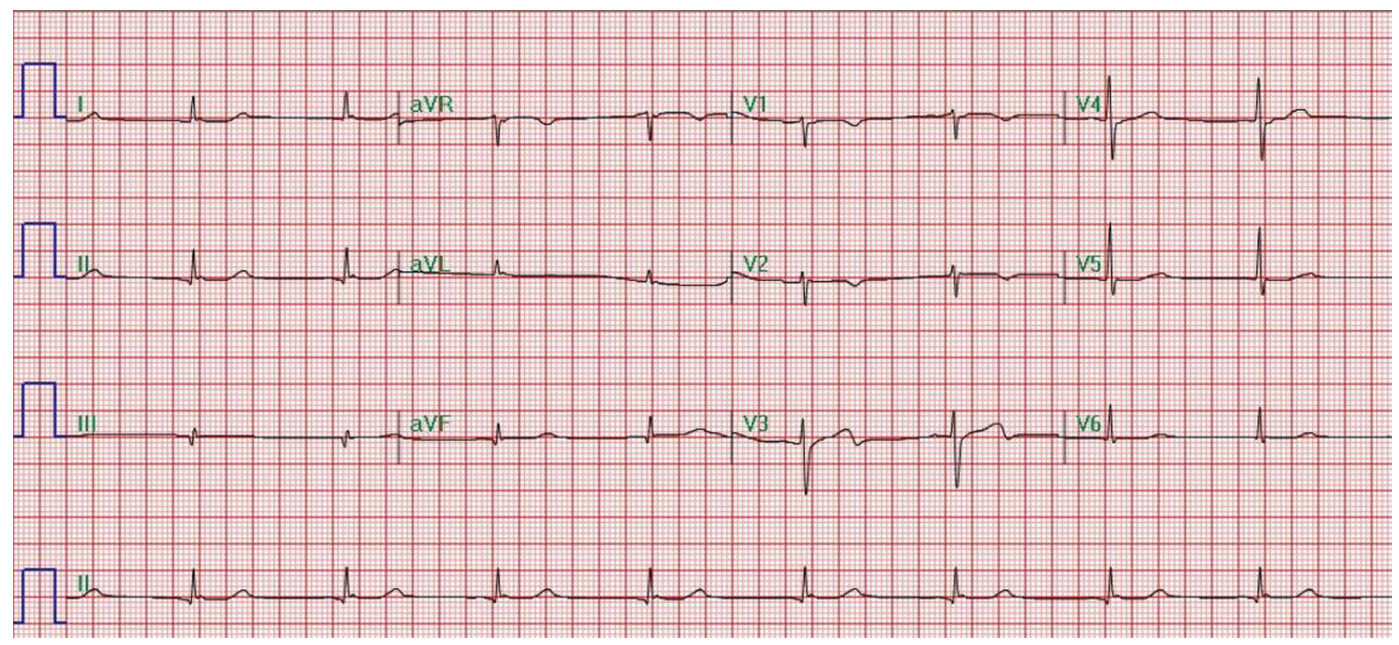

Figure 1. Normal sinus bradycardia.

three times per week. However, his blood urea nitrogen and serum creatinine levels still failed to improve, and he remained in a state of metabolic acidosis. On day 14 of his admission, the patient's condition deteriorated $2 \mathrm{~h}$ in dialysis treatment. He lost his gag and cough reflexes, and his pupils became dilated and fixed. A repeat head CT scan was performed which showed evidence of brain herniation. The brain herniation was confirmed using brain scan flow which showed empty bulb

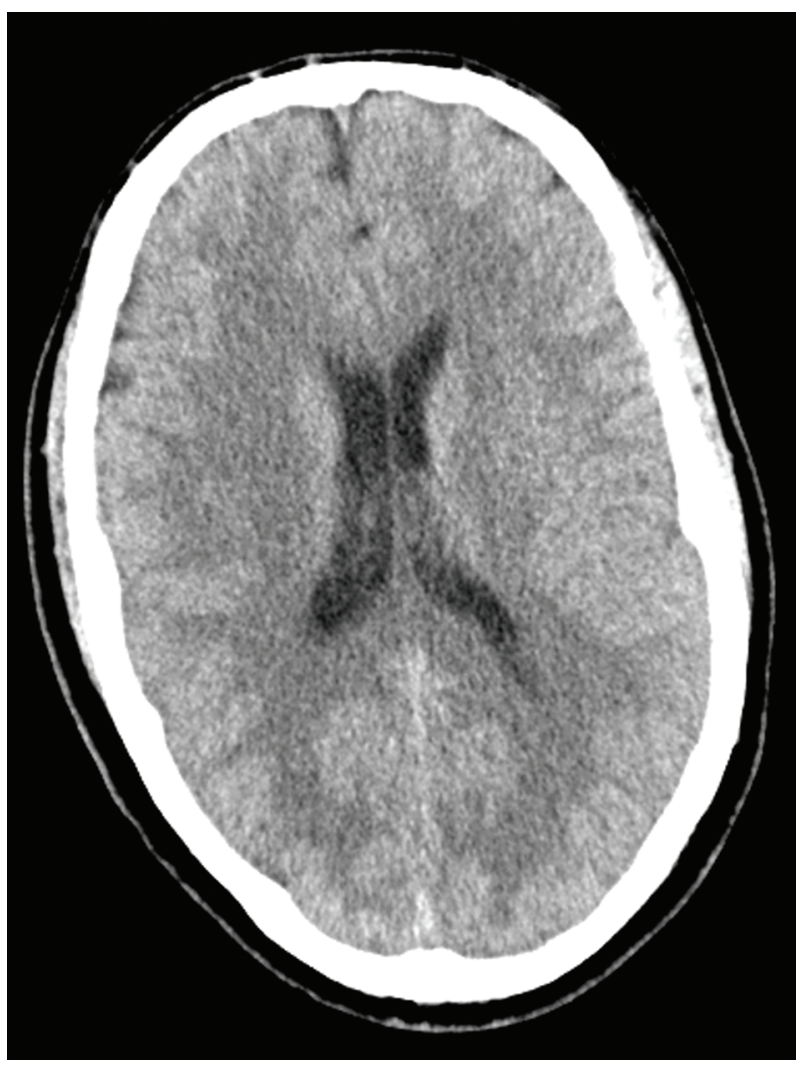

Figure 2. Computed tomography (CT) of the head was negative for intracranial bleed and did not show any anoxic encephalopathy. sign (Fig. 3). Repeat lab investigations conducted immediately following hemodialysis revealed a $\mathrm{pH}$ of $7.24, \mathrm{HCO}_{3}$ of 26 , sodium of $134 \mathrm{mmol} / \mathrm{L}$, potassium $3.0 \mathrm{mmol} / \mathrm{L}$, urea of $89 \mathrm{mg} /$ $\mathrm{dL}$, and serum creatinine of $11.2 \mathrm{mg} / \mathrm{dL}$. Ultimately, the patient was pronounced brain dead the patient's family opted for organ donation.

\section{Discussion}

DDS is a rare, but serious disease, with fatal complications including cerebral edema and brain death. DDS occurs during or after dialysis [1]. Risk factors for DDS include dialysis treatment, markedly elevated blood urea concentration, predialysis (i.e. $>175 \mathrm{mg} / \mathrm{dL}$ or $60 \mathrm{mmol} / \mathrm{L}$ ), chronic kidney disease (CKD as compared with acute kidney injury (AKI)), severe metabolic acidosis, older age, pediatric patients, pre-existing neurologic disease (head trauma, stroke, seizure disorder), conditions characterized by cerebral edema (hyponatremia, hepatic encephalopathy, malignant hypertension), and any condition that increases the permeability of the blood brain barrier (sepsis, vasculitis, thrombotic thrombocytopenic purpura-hemolytic uremic syndrome, encephalitis, or meningitis) [2-6]. DDS can present with a wide spectrum of clinical manifestations, both mild and severe. Mild symptoms of DDS include nausea, vomiting [1], headache, anorexia, blurred vision, muscle cramps, disorientation, restlessness, hypertension and dizziness. More severe symptoms of DDS are consistent with central nervous system dysfunction, such as seizures, central pontine myelinolysis, and ultimately coma and death. DDS has been credited for acute electroencephalographic abnormalities and structural changes on diagnostic imaging following rapid hemodialysis.

There are three theories proposed to explain the pathogenesis of DDS: the reverse urea effect, idiogenic osmoles hypothesis, and paradoxical brain acidosis. Kennedy et al first suggested the reverse urea effect after it was noted in a mouse model that urea was removed more slowly from cerebral spinal fluid than from blood during intermittent renal replacement 


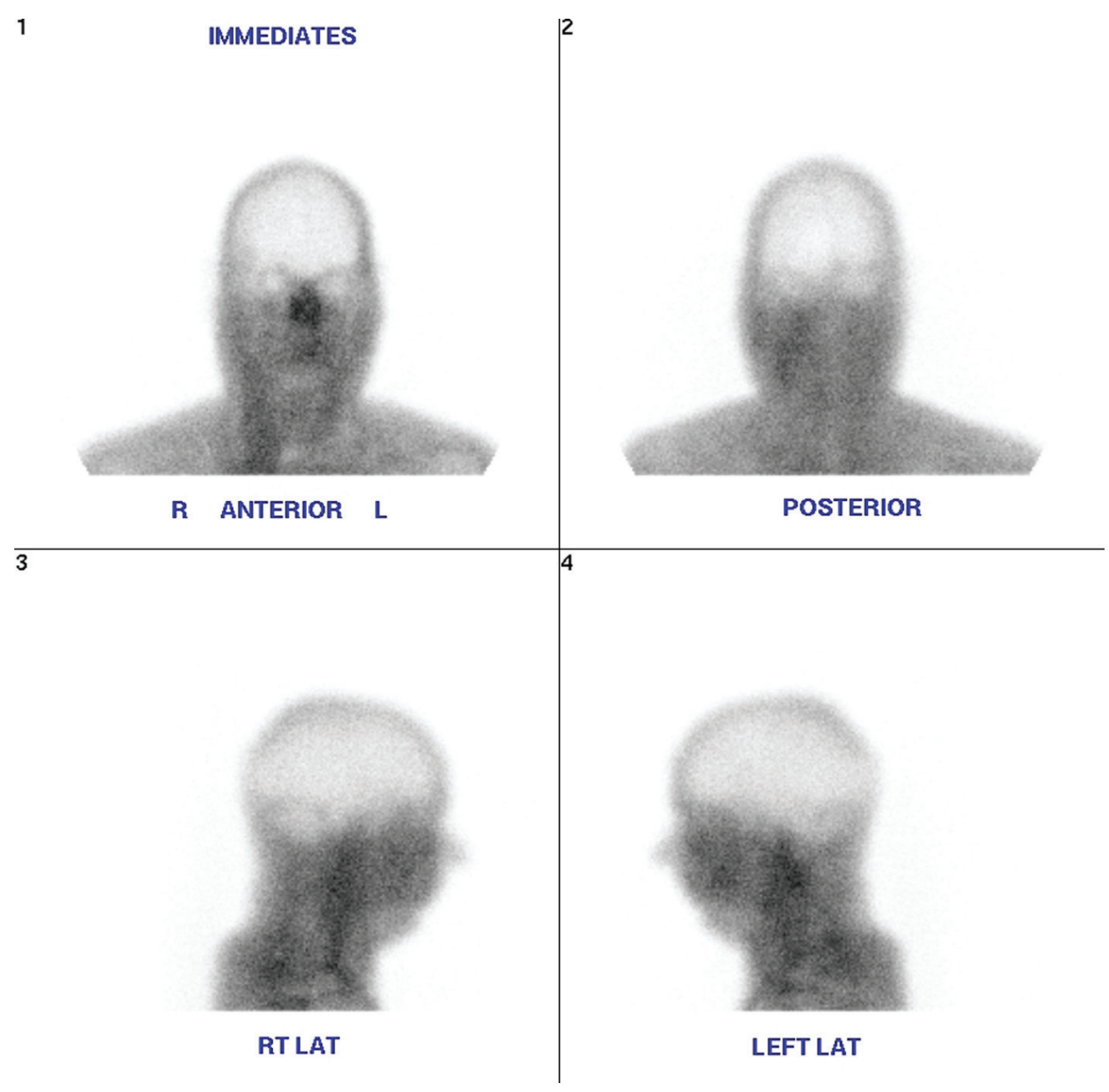

Figure 3. Brain scan flow which showed empty bulb sign.

therapy (IRRT) [7]. IRRT leads to rapid reduction in plasma urea with delay in the brain urea clearance creating an osmotic gradient between the plasma and brain $[8,9]$. The discovery of urea transporters further supported the reverse urea effect because these transporters are down regulated in uremic states, thereby decreasing the ability of brain to quickly adapt to the plasma urea loss.

The idiopathic osmoles hypothesis suggests that dialysis may generate other, but thus far unidentified, osmotically active molecules that contribute to brain edema [10].

Finally, paradoxical brain acidosis suggests that as renal replacement therapy (RRT) corrected the acidosis, brain $\mathrm{pH}$ decreased, thereby creating an osmotic gradient by displacement of intracellularly protein bound potassium and sodium. Consequently, these ions become osmotically active causing influx of water [11].

In our case patient had risk factors like severe sepsis, septic shock, increased BUN, and severe metabolic acidosis, which have altered the blood brain barrier and may have contributed for the development of DDS.

As DDS is the development of an osmotic gradient causing water to move into the brain, preventing the development of this gradient should prevent the syndrome. The simplest way to do this is to perform hemofiltration on the patient instead of dialysis. This method of treatment relies on the convective removal of solute from the patient in place of diffusive removal. Thus, the osmolalities of the body fluid compartments will not change as rapidly as they do during standard hemodialysis. This method was shown to reduce some of the symptoms that are related to the disequilibrium syndrome.

\section{Conclusion}

If a patient with severe sepsis and septic shock and with acute kidney failure who needs dialysis may develop DDS even after repeated sessions of the hemodialysis. DDS might have contributed to sudden deterioration and death in this young patient with septic shock.

\section{Competing Interests}

The authors declare that there is no conflict of interests regarding the publication of this manuscript. This is to state that there has been no activity or involvements that will raise the question of bias in this case report or any of the conclusions or opinions that it stands for. 


\section{References}

1. Shaikh N, Louon A, Hanssens Y. Fatal dialysis disequilibrium syndrome: A tale of two patients. J Emerg Trauma Shock. 2010;3(3):300.

2. Arieff AI. Dialysis disequilibrium syndrome: current concepts on pathogenesis and prevention. Kidney Int. 1994;45(3):629-635.

3. Zepeda-Orozco D, Quigley R. Dialysis disequilibrium syndrome. Pediatr Nephrol. 2012;27(12):2205-2211.

4. Patel N, Dalal P, Panesar M. Dialysis disequilibrium syndrome: a narrative review. Semin Dial. 2008;21(5):493498.

5. Bagshaw SM, Peets AD, Hameed M, Boiteau PJ, Laupland KB, Doig CJ. Dialysis Disequilibrium Syndrome: brain death following hemodialysis for metabolic acidosis and acute renal failure--a case report. BMC Nephrol. 2004;5:9.
6. Marshall MR, Golper TA. Low-efficiency acute renal replacement therapy: role in acute kidney injury. Semin Dial. 2011;24(2):142-148.

7. Kennedy A, Linton A, Eaton J. Urea levels in cerebrospinal fluid after hemodialysis. The Lancet. 1962;279(7226):410411.

8. Silver SM, DeSimone JA, Jr., Smith DA, Sterns RH. Dialysis disequilibrium syndrome (DDS) in the rat: role of the "reverse urea effect". Kidney Int. 1992;42(1):161-166.

9. Rosen SM, O'Connor K, Shaldon S. Haemodialysis Disequilibrium. Br Med J. 1964;2(5410):672-675.

10. Arieff AI, Massry SG, Barrientos A, Kleeman CR. Brain water and electrolyte metabolism in uremia: effects of slow and rapid hemodialysis. Kidney Int. 1973;4(3):177187.

11. Arieff AI, Guisado R, Massry SG, Lazarowitz VC. Central nervous system $\mathrm{pH}$ in uremia and the effects of hemodialysis. J Clin Invest. 1976;58(2):306-311. 